\title{
Prognostic factors for lung function in systemic sclerosis: prospective study of
}

\section{5 cases}

\author{
M. Gilson*, D. Zerkak*, J. Wipff*, D. Dusser", A.T. Dinh-Xuan ${ }^{\star}$, V. Abitbol ${ }^{+}$, \\ S. Chaussade ${ }^{+}$, P. Legmann ${ }^{\S}$, A. Kahan* and Y. Allanore*
}

ABSTRACT: The aims of the present study were to identify prognostic factors for systemic sclerosis (SSc)-related interstitial lung disease and to clarify the possible causative role of manometric oesophageal involvement.

Consecutive SSc patients underwent pulmonary function tests and oesophageal manometry. They were included in the study if pulmonary function tests were repeated $>12$ months after baseline. The primary end-point was a decrease of $\geqslant 10 \%$ of the predicted value in forced vital capacity (FVC). The secondary end-points were a decrease of $\geqslant 15 \%$ pred in lung carbon monoxide diffusing capacity (DL,CO) and a decrease of $\geqslant 20 \%$ pred in FVC.

Of the 105 patients (45 diffuse SSc; median disease duration $2.0 \mathrm{yrs}$ ), 23 (23\%) had a FVC of $<80 \%$ pred, 60 (59\%) had a DL,CO of $<80 \%$ pred and $57(54 \%)$ showed severe oesophageal hypomotility at baseline. Over $72 \pm 46$ months, 29 (28\%) patients displayed a decrease of $\geqslant 10 \%$ pred in FVC, 39 (40\%) of 98 patients displayed DL,CO decline and 19 (18\%) patients displayed a decrease of $\geqslant 20 \%$ pred in FVC. On multivariate analysis, diffuse SSc was a significant predictor for a decrease of $\geqslant 10 \%$ pred in FVC $(p=0.01)$. No other predictor of a decrease in pulmonary function was identified.

Only diffuse SSc was predictive of a decrease in pulmonary function in this early-SSc cohort. This does not support preliminary data suggestive of a causative role of oesophageal involvement.

KEYWORDS: Diffuse scleroderma, gastro-oesophageal reflux, interstitial lung disease, oesophageal manometry, prognostic factors, systemic sclerosis

I nterstitial lung disease (ILD) occurs in up to $75 \%$ of patients with systemic sclerosis (SSc) [1], but progresses to severe restrictive lung disease in only $\sim 13 \%$ of these patients [2]. Pulmonary volume seems to decrease principally in the first 4 yrs of the disease [2]. In recent series, ILD has been identified as one of the leading causes of death in SSc $[3,4]$, being implicated in $\sim 30 \%$ of SScrelated deaths [3]. The therapeutic management of this condition, therefore, remains a major challenge. Studies assessing the efficacy of immunosuppressive drugs, such as cyclophosphamide, for the treatment of SSc-related ILD (SSc-ILD) have mostly generated disappointing results [5]. In particular, a recent randomised controlled trial failed to demonstrate clearly that oral cyclophosphamide treatment for $1 \mathrm{yr}$ was effective, showing only a modest increase in forced vital capacity (FVC) at 12 months [6], which was no longer detectable at 24 months [7]. These results may be accounted for by the lack of identification of a subgroup of SSc patients at high risk of pulmonary progression [5].

A possible causative role of gastro-oesophageal reflux (GOR) in promoting idiopathic lung fibrosis $[8,9]$, as well as SSc-ILD [10-12], was raised by previous studies. In particular, MARIE et al. [11] suggested that the level of gastro-oesophageal dysmotility was associated with the frequency and severity of pulmonary involvement. However, conflicting results have been obtained in other studies [13, 14]. Indeed, in a previous crosssectional study investigating the prevalence of Barrett's oesophagus in the present cohort of SSc patients, no significant association was found between gastro-oesophageal involvement and ILD, assessed by both computed tomography (CT) and pulmonary function tests (PFTs) [14].

In the present study, the possible link between SScILD and manometric oesophageal involvement,

\section{AFFILIATIONS}

Depts of *Rheumatology A,

\#Pneumology,

"Respiratory Physiology,

${ }^{+}$Gastroenterology, and

${ }^{\S}$ Radiology A, Paris Descartes

University, Cochin Hospital, Paris, France.

CORRESPONDENCE

Y. Allanore

Université Paris Descartes, Service

de Rhumatologie A

Hôpital Cochin

27 rue du faubourg St-Jacques

75014 Paris

France

E-mail: yannick.allanore@

cch.aphp.fr

Received:

April 102009

Accepted after revision:

June 082009

First published online:

June 182009 
and the prognostic factors for a decrease in pulmonary function, were investigated using a prospective follow-up design.

\section{PATIENTS AND METHODS}

The present cohort of SSc patients has been described elsewhere [14]. The patients included in this previous study and new patients attending consultations in the Dept of Rheumatology A (Paris Descartes University, Cochin Hospital, Paris, France) up to January 2006 were included in a prospective follow-up study following baseline evaluation of oesophageal and systemic involvement. The inclusion criteria were oesophageal manometry and PFTs at baseline, and repeated PFTs $>12$ months after the initial assessment. The prospective data were collected according to routine care assessments based on at-least annual systemic follow-up. The exclusion criteria were being lost to follow-up, assessment only once at Cochin Hospital, and no repeat PFTs or $<1 \mathrm{yr}$ of follow-up data available. All patients gave informed consent and the study was approved by the local ethics committee (Ile-de-France Committee for the Protection of Persons III, Tarnier Hospital, Paris, France).

The end of the study period corresponded, for each patient, to the last admission for routine follow-up, including PFTs, available in January 2008. The treatments administered during the study period that might interfere with the study results are as follows: proton-pump inhibitors (PPIs), oral or intravenous cyclophosphamide treatment for $\geqslant 6$ months, and steroids (intravenous or oral at a dose of $>10 \mathrm{mg} \cdot$ day $^{-1}$ prednisoneequivalent). The overall severity of the disease was assessed using the revised Medsger scale [15]. An increase in Medsger scale class between baseline and the end of the study was interpreted as an overall deterioration of the disease.

All patients underwent oesophageal manometry at baseline, as previously described [14]. As in MARIE et al. [11], oesophageal motility patterns were classified in four stages according to Hurwitz's classification [16]. Hurwitz stage 1 corresponds to normal oesophageal motility; stage 2 corresponds to uncoordinated peristalsis with normal pressure wave amplitude; stage 3 corresponds to uncoordinated peristalsis with low pressure wave amplitude; and stage 4 corresponds to aperistalsis and decreased lower oesophageal sphincter (LOS) pressure. Severe oesophageal hypomotility was defined as Hurwitz stage 3 or 4 .

PFTs were carried out systematically at baseline, and the last repeat PFT defined the end of the follow-up period. PFT results are expressed as a percentage of the standardised predicted value for age, sex and height. Restrictive lung disease was defined as an FVC of $<80 \%$ pred, and severe restrictive lung disease was defined as an FVC of $\leqslant 50 \%$ pred [2].

As in previous studies assessing functional deterioration in SSc-ILD [17], the present end-points included a decrease of $\geqslant 10 \%$ pred in FVC over the study period, which was the principal end-point (FVC decline), and a decrease of $\geqslant 15 \%$ pred in lung carbon monoxide diffusing capacity (DL,CO), which was a secondary end-point ( $D \mathrm{~L}, \mathrm{CO}$ decline). Another secondary end-point was a decrease of $\geqslant 20 \%$ pred in FVC (severe FVC decline).

The present study was longitudinal and observational. The associations between the baseline levels of the variables studied and the three predefined end-points were evaluated after the end of the prospective follow-up period. Univariate and multivariate analyses were carried out. Differences between groups with a $\mathrm{p}$-value of $\leqslant 0.05$ were selected for multivariate analysis. Survival was evaluated using the Kaplan-Meier method. Log-rank tests were used to assess differences in survival probabilities. Cox's regression analysis was used to evaluate the association between baseline values and pulmonary outcome. Significance was assumed if the null hypothesis could be rejected at $\mathrm{p}<0.05$. Statistical analysis was performed with $R_{\mathbb{B}}$ software (version 2.5.0 for Windows; Lucent Technologies, Paris, France).

During the study period, $47(44.5 \%)$ patients underwent two routine CT scans separated by $\geqslant 24$ months at Cochin Hospital, based on the physician's decision. An additional study assessing the concordance between PFT and CT scan outcomes was carried out for this subgroup. Only high-resolution CT scans performed in the radiology unit of Cochin Hospital were considered for analyses. All CT scans were assessed by the same trained radiologist, blinded to the patient's clinical condition and the temporal order of the CT scans for each patient, using a grading system described by Wells et al. [18]. Grade 0 corresponds to normal HRCT results; grade 1 corresponds to predominant areas of ground-glass attenuation; grade 2 corresponds to equally extensive areas of ground-glass attenuation and reticulation; and, for grade 3 , reticulations, microcystic spaces and bronchiectasis are predominant. It was concluded that deterioration had occurred in cases in which the final CT score was higher than baseline score. The kappa coefficient was calculated to assess the concordance between primary outcome and CT scan deterioration.

\section{RESULTS}

The baseline characteristics of the 105 SSc patients recruited are reported in table 1 , and detailed for each cutaneous subtype of the disease.

After a mean follow-up duration of $71.8 \pm 46.4$ months, 36 (35\%) patients displayed an overall deterioration (table 2). At baseline, $13 \%$ were receiving nonsteroidal anti-inflammatory drugs and $24 \%$ prokinetics. All of the patients received daily treatment with PPIs throughout the study period. No patient received intravenous steroids or oral steroids at a dose of $>10 \mathrm{mg} \cdot$ day $^{-1}$ prednisone-equivalent. No patient was treated with oral cyclophosphamide during the study period, but four $(4 \%)$ patients received intravenous cyclophosphamide at a dose of $0.7 \mathrm{~g} \cdot \mathrm{m}^{-2}$ monthly for $\geqslant 6$ months for the treatment of severe and progressive ILD.

Two FVCs (the first end-point) were available for all patients. Two DL,CO (baseline and follow-up) were not available for seven patients, either because the restrictive lung disease was too severe $(n=4)$ or due to technical problems $(n=3)$. Two DL,CO (the secondary end-point) were available for $98(93 \%)$ patients. PFT outcome in the overall cohort is shown in table 2, and detailed for each cutaneous subtype. Of the four patients treated with intravenous cyclophosphamide, three displayed both a severe FVC decline and DL,CO decline; the remaining patient displayed no decline in FVC or DL,CO.

On univariate analysis, anti-topoisomerase I and the diffuse cutaneous SSc (dcSSc) subtype were associated with FVC 


\begin{tabular}{|c|c|c|c|c|}
\hline \multirow[t]{2}{*}{ TABLE 1} & \multicolumn{4}{|c|}{$\begin{array}{l}\text { Baseline demographic characteristics, } \\
\text { pulmonary function test results and oesophageal } \\
\text { profiles by systemic sclerosis (SSc) subtype }\end{array}$} \\
\hline & & Total & dcSSc & IcSSc \\
\hline \multicolumn{2}{|l|}{ Subjects $\mathrm{n}$} & 105 & 45 & 60 \\
\hline \multicolumn{2}{|l|}{ Age yrs } & $52.7 \pm 11.8$ & $52.2 \pm 13.4$ & $53.1 \pm 10.6$ \\
\hline \multicolumn{2}{|l|}{ Female } & $90(85.7)$ & $33(73.3)$ & $57(95.0)$ \\
\hline \multicolumn{2}{|c|}{ Black } & $8(7.6)$ & $5(10.9)$ & $3(5.1)$ \\
\hline \multicolumn{2}{|c|}{ SSc duration yrs $\#$} & $2.0(0.0-5.0)$ & $2.0(1.0-5.0)$ & $1.0(0.0-4.0)$ \\
\hline \multicolumn{2}{|c|}{ Prior digital ulcer history } & $32(30.5)$ & $18(39.1)$ & $14(23.7)$ \\
\hline \multicolumn{2}{|c|}{$\mathbf{P A H}^{\circ}$} & $1(0.9)$ & $1(2.2)$ & $0(0)$ \\
\hline \multicolumn{2}{|c|}{ Medsger stage $\geqslant 3^{+}$} & $15(14.4)$ & $8(17.4)$ & $7(11.9)$ \\
\hline \multicolumn{2}{|c|}{ ANA-positive $^{\S}$} & $89(84.8)$ & 40 (88.9) & $49(81.7)$ \\
\hline \multicolumn{2}{|c|}{ Anti-topoisomerase I } & $30(28.5)$ & $30(65.2)$ & $0(0)$ \\
\hline \multicolumn{2}{|c|}{ Anticentromere antibody } & $21(21.0)$ & $0(0)$ & 21 (35.6) \\
\hline \multicolumn{2}{|c|}{ Anti-ribonucleoprotein } & $8(7.6)$ & $3(6.7)$ & $5(8.3)$ \\
\hline \multicolumn{2}{|c|}{ FVC $<80 \%$ pred } & 23 (22.9) & 15 (33.3) & $8(13.3)$ \\
\hline \multicolumn{2}{|c|}{ FVC $<70 \%$ pred } & $7(6.7)$ & $5(10.9)$ & $2(3.4)$ \\
\hline \multicolumn{2}{|c|}{ FVC $<50 \%$ pred } & $0(0)$ & $0(0)$ & $0(0)$ \\
\hline \multicolumn{2}{|c|}{$D$ L,CO $<80 \%$ pred $^{f}$} & $60(58.8)$ & $34(73.9)$ & $26(44.1)$ \\
\hline \multicolumn{2}{|c|}{ Abn oesophageal manometry } & 80 (76.2) & 40 (88.9) & $40(66.7)$ \\
\hline \multicolumn{2}{|c|}{ Hurwitz stage $\geqslant 3^{+}$} & $57(54.6)$ & $30(65.2)$ & $27(45.8)$ \\
\hline \multicolumn{2}{|c|}{ Oesophageal aperistalsis } & $38(36.2)$ & $21(45.6)$ & $17(28.8)$ \\
\hline \multicolumn{2}{|c|}{ LOS pressure $<10 \mathrm{mmHg}$} & $52(49.5)$ & $26(57.7)$ & $26(43.3)$ \\
\hline
\end{tabular}

Data are presented as mean $\pm \mathrm{SD}, \mathrm{n}$ (\%) or median (interquartile range), unless otherwise indicated. dcSSc: diffuse cutaneous SSc; IcSSc: limited cutanous SSc; PAH: pulmonary arterial hypertension; ANA: antinuclear antibody; FVC: forced vital capacity; \% pred: \% predicted; DL,CO: diffusing capacity of the lung for carbon monoxide; Abn: abnormal; LOS: lower oesophageal sphincter. \#: from diagnosis of first non-Raynaud symptom; ": pre-capillary PAH diagnosed by right heart catheterisation $(n=101) ;{ }^{+}: n=104$, diagnosed by histology; ${ }^{\text {s: }}$ dilution of more than $1: 80 ; f: n=102$.

decline $(p=0.009$ and $p=0.001$, respectively) and severe FVC decline ( $p=0.02$ for both) (table 3$)$. Baseline FVC of $<70 \%$ pred was another candidate predictor $(p=0.04)$, but caution is required in the interpretation of this result since only seven patients had a baseline FVC of $<70 \%$ pred. No association with FVC outcome was observed for the other variables considered: sex, ethnicity, age, SSc duration, and oesophageal involvement. The results of the multivariate analysis are shown in table 4 . In the models concerning FVC decline, only dcSSc was a significant predictor, irrespective of SSc duration and baseline FVC. In the model concerning severe FVC decline, no significant predictor was identified.

On univariate analysis, two oesophageal variables were identified as potential predictors of DL,CO decline, severe oesophageal hypomotility $(p=0.009)$ and aperistalsis $(p=0.02)$ (table 5). However, these factors were nonsignificant on multivariate analysis: hazard ratio (HR) 2.10 (95\% confidence interval (CI) 0.75-5.84), $\mathrm{p}=0.15$; and HR 1.23 (95\% CI 0.48-3.16), $\mathrm{p}=0.66$, respectively. Therefore, it was not possible to identify a predictor of $\mathrm{DL}, \mathrm{CO}$ decline in the present cohort.

The whole population of SSc patients underwent at least one CT scan during the study, CT-proven ILD was found in 52 out

\begin{tabular}{|c|c|c|c|}
\hline \multicolumn{4}{|c|}{$\begin{array}{l}\text { Overall and pulmonary outcomes during the } \\
\text { study period }{ }^{\#} \text { by systemic sclerosis (SSc) } \\
\text { subtype }\end{array}$} \\
\hline & Total & dcSSc & IcSSc \\
\hline Subjects $n$ & 105 & 45 & 60 \\
\hline \multicolumn{4}{|l|}{ Final PFT results } \\
\hline FVC $<80 \%$ pred & $34(32.4)$ & $23(50.0)$ & $10(16.9)$ \\
\hline FVC $\leqslant 50 \%$ pred & $9(8.5)$ & $9(19.6)$ & $0(0.0)$ \\
\hline$D \mathrm{~L}, \mathrm{CO}<80 \%$ pred & $81(82.7)$ & $33(71.7)$ & $48(31.4)$ \\
\hline \multicolumn{4}{|l|}{ PFT outcome } \\
\hline FVC decline $\geqslant 10 \%$ pred & $29(27.6)$ & $21(45.7)$ & $8(13.6)$ \\
\hline FVC decline $\geqslant 20 \%$ pred & $19(18.1)$ & $13(28.3)$ & $6(10.2)$ \\
\hline DL,CO decline $\geqslant 15 \%$ pred & $39(39.8)$ & $14(30.4)$ & $25(42.4)$ \\
\hline $\begin{array}{l}\text { FVC decline } \geqslant 10 \% \text { pred or } D L, C O \\
\text { decline } \geqslant 15 \% \text { pred }\end{array}$ & $55(52.4)$ & $26(56.5)$ & $29(49.2)$ \\
\hline \multicolumn{4}{|l|}{ Overall severity at end of follow-up ${ }^{+}$} \\
\hline Final Medsger stage $\geqslant 3$ & $39(37.5)$ & $25(54.3)$ & $14(23.7)$ \\
\hline Overall aggravation of SSc & $36(34.6)$ & $22(27.8)$ & $14(23.7)$ \\
\hline \multicolumn{4}{|c|}{$\begin{array}{l}\text { Data are presented as } n(\%) \text {, unless otherwise indicated. dcSSc: diffuse } \\
\text { cutaneous SSc; IcSSc: limited cutaneous SSc; PFT: pulmonary function test; } \\
\text { FVC: forced vital capacity; \% pred: \% predicted; DL,CO: diffusing capacity of the } \\
\text { lung for carbon monoxide. }{ }^{\#}: 71.8 \pm 46.4 \text { months; }{ }^{\bullet}: n=9 \text {; }^{+}: n=104 \text {. }\end{array}$} \\
\hline
\end{tabular}

of 105 patients, and all patients with FVC decline had ILD. In the subgroup of patients included in the study comparing PFT and CT scan outcomes (two CT scans separated by $\geqslant 24$ months performed in Cochin Hospital; $n=47$ ), the mean interval between CT scans was $45.8 \pm 25.8$ months. CT scan scores at baseline were distributed as follows: grade $0, \mathrm{n}=16$ $(34 \%)$; grade $1, n=16(34 \%)$; grade $2, n=4(9 \%)$; and grade 3 , $n=11(23 \%)$. Eight patients displayed deterioration on CT. Fifteen patients displayed a decline in FVC during the study period. The kappa coefficient for correlation between CT scan deterioration and FVC decline was 0.6, demonstrating reasonably good concordance between the two methods.

\section{DISCUSSION}

The present study was designed to evaluate predictors of pulmonary function deterioration in nonselected SSc patients undergoing complete oesophageal assessment at baseline. This is the largest prospective study aimed at identification of a potential link between oesophageal involvement and ILD course. The dcSSc subtype was the only significant predictor of poor pulmonary outcome identified, irrespective of SSc duration, baseline FVC and overall deterioration of the disease. As decreased FVC in SSc may be related to stiff chest wall and diaphragmatic dysfunction, an additional analysis was performed comparing PFT and CT scan outcomes in approximately half of the patients. It was demonstrated that the two methods of assessment of ILD were reasonably well correlated in the present cohort, as previously observed [19]. Moreover, the frequency of FVC decline in this subgroup was similar to that in all patients, suggesting that this subgroup was representative of the entire cohort.

In SSc, the loss of oesophageal peristalsis results from both fibrosis and vasculopathy, affecting oesophageal smooth 


\begin{tabular}{|c|c|c|c|c|}
\hline \multirow[t]{2}{*}{ TABLE 3} & \multicolumn{4}{|c|}{$\begin{array}{l}\text { Baseline characteristics in patients with and } \\
\text { without forced vital capacity (FVC) decline during } \\
\text { follow-up }{ }^{\#} \text { : univariate analysis }\end{array}$} \\
\hline & & Decline & No decline & $\mathrm{p}$-value \\
\hline \multicolumn{2}{|c|}{ Subjects $n$} & 29 & 76 & \\
\hline \multicolumn{2}{|c|}{ Abn oesophageal manometry } & $27(93.1)$ & $53(69.7)$ & 0.06 \\
\hline \multicolumn{2}{|c|}{ LOS pressure $<10 \mathrm{mmHg}$} & $17(58.6)$ & $35(46.1)$ & 0.81 \\
\hline \multicolumn{2}{|c|}{ Aperistalsis } & $16(55.2)$ & $21(27.6)$ & 0.23 \\
\hline \multicolumn{2}{|c|}{ Hurwitz stage $\geqslant 3$} & $21 / 28(75.0)$ & $36(47.4)$ & 0.08 \\
\hline \multicolumn{2}{|c|}{ Hurwitz stage 4} & $11(37.9)$ & $17(22.4)$ & 0.81 \\
\hline \multicolumn{2}{|c|}{ Baseline FVC $<75 \%$ pred } & $5(17.2)$ & $11(14.5)$ & 0.42 \\
\hline \multicolumn{2}{|c|}{ Baseline FVC $<70 \%$ pred } & $2(6.8)$ & 5 (6.6) & 0.04 \\
\hline \multicolumn{2}{|c|}{ SSc duration yrs } & $2.0(0.0-3.0)$ & $1.5(0.0-5.2)$ & 0.20 \\
\hline \multicolumn{2}{|c|}{ Anti-topoisomerase I } & $15(51.7)$ & $15(19.7)$ & 0.009 \\
\hline \multicolumn{2}{|c|}{ Diffuse cutaneous SSc } & $21(72.4)$ & $24(31.6)$ & 0.001 \\
\hline \multicolumn{2}{|c|}{ Black } & $4(13.8)$ & $4(5.3)$ & 0.60 \\
\hline \multicolumn{2}{|c|}{ Male } & $5(17.2)$ & $10(13.2)$ & 0.42 \\
\hline \multicolumn{2}{|c|}{ Baseline age $>50 \mathrm{yrs}$} & $13(44.8)$ & $45(59.2)$ & 0.65 \\
\hline \multicolumn{2}{|c|}{ Overall aggravation of SSc } & $15(51.7)$ & $21(27.6)$ & 0.26 \\
\hline
\end{tabular}

muscle and its innervation [20] and frequently leading to severe GOR. Some studies have suggested that GOR may contribute to idiopathic pulmonary fibrosis $[8,9]$ and SSc-ILD [10-12] through repeated microaspiration of gastric content into the respiratory tract. Other arguments supporting this hypothesis are the preferential localisation of SSc-ILD in the lower lung zones [19] and the frequent centrilobular localisation of fibrosis in SSc patients with GOR [21]. However, the preliminary studies provide a low level of evidence due to their cross-sectional design or short follow-up periods, small numbers of patients and, in some studies, absence of multivariate analysis. For example, MARIE et al. [11] carried out a cross-sectional study in 43 patients, with a longitudinal study over 2 yrs in only 18 of them and univariate analysis alone. Moreover, they identified an association between GOR and $D \mathrm{~L}, \mathrm{CO}$, which depended on both ILD and vasculopathy, but no correlation with FVC, which better reflects ILD. More recently, in a cohort of 40 patients, SAVARINO et al. [12] identified greater oesophageal acid exposure (assessed by 24-h impedance $\mathrm{pH}$ monitoring) in SSc patients with ILD than in those without ILD. The design of this study differs from that of the present study as it was cross-sectional, whereas the present study was prospective. SAVARINO et al. [12] identified some factors associated with ILD, including reflux episodes. However, only univariate analyses were provided, thus not permitting estimation of the weight of the reflux among various associated factors. In addition, SSc patients had to stop PPIs before their study; this increases the role of reflux during PPI therapy since the large majority of SSc patients receive long-term therapy with these drugs. Nevertheless, one of the important points in this study was the demonstration of the presence of non-acid reflux and also the proximal extent of reflux episodes [12]. This

\begin{tabular}{|c|c|c|c|}
\hline \multirow[t]{2}{*}{ TABLE 4} & \multicolumn{3}{|c|}{$\begin{array}{l}\text { Multivariate analysis assessing candidate } \\
\text { predictors of decrease in forced vital capacity } \\
\text { (FVC) during follow-up\# }\end{array}$} \\
\hline & & HR $(95 \% \mathrm{Cl})$ & $\mathrm{p}$-value \\
\hline \multicolumn{4}{|c|}{ FVC decline $\geqslant 10 \%$ pred } \\
\hline \multicolumn{4}{|c|}{ First model } \\
\hline Anti-topo & omerase I & $0.92(0.33-2.57)$ & 0.88 \\
\hline Diffuse $c$ & aneous SSc & $4.22(1.37-13.00)$ & 0.009 \\
\hline Baseline & VC $<75 \%$ pred & $0.60(0.21-1.71)$ & 0.33 \\
\hline SSc dura & & $0.61(0.25-1.50)$ & 0.28 \\
\hline \multicolumn{4}{|c|}{ Second model } \\
\hline Anti-topo & omerase I & $0.99(0.36-2.72)$ & 0.99 \\
\hline Diffuse $c$ & aneous SSc & $3.41(1.15-10.08)$ & 0.02 \\
\hline Baseline & VC $<70 \%$ pred & $3.08(0.62-15.20)$ & 0.16 \\
\hline \multicolumn{4}{|c|}{ FVC decline $\geqslant 20 \%$ pred } \\
\hline Anti-topoisc & nerase I & $1.54(0.40-5.87)$ & 0.52 \\
\hline Diffuse cuta & eous SSc & $2.24(0.54-9.24)$ & 0.26 \\
\hline
\end{tabular}

Where $95 \%$ confidence intervals (Cls) are unavailable, hazard ratios (HRs) alone are shown. \% pred: \% predicted; SSc: systemic sclerosis. ${ }^{\#}: 71.8 \pm 46.4$ months. Data in bold are significant.

suggests that reflux-reducing, and not only acid-suppressive, therapies should be included in future studies aimed at the evaluation of the influence of oesophageal involvement on ILD outcomes.

In the present study, oesophageal involvement was assessed with a different method from that of SAVARINO et al. [12]. Manometry is considered to be one of the most accurate methods of investigating oesophageal motility [22]. The prevalence of abnormal oesophageal manometric findings was similar to that previously reported for SSc patients $(80 \%$ in the study of BASSOTTI et al. [23]), as was mean LOS pressure (15.8 $\mathrm{mmHg}$ according to YARZE et al. [24]). Some studies have shown that oesophageal acid exposure in patients with low levels of oesophageal motility may be better correlated with peristaltic abnormalities than with LOS pressure [25]. For this reason, Hurwitz's criteria, based on oesophageal motility, were used to assess oesophageal impairment.

In the present cohort, no correlation between PFT result deterioration and oesophageal impairment was highlighted, taking into account both peristalsis and LOS pressure. SSc is a multifactorial disease, in which severe impairment tends to occur in several organs simultaneously [17]. Consequently, GOR is probably a confounding factor rather than a cause of ILD. The preferential localisation of SSc-ILD in the lower lung zones [19] may instead be accounted for by the large number of vessels in these areas; indeed, vasculopathy is thought to play a major role in the pathogenesis of lung fibrosis [26].

The main risk factors for the occurrence of ILD in SSc identified to date are the dcSSc subtype, high skin fibrosis score and antitopoisomerase I [17]. In the Scleroderma Lung Study (SLS) [27], which was designed to assess the efficacy of cyclophosphamide for the treatment of SSc-ILD, similar baseline PFT profiles and outcome were observed in the limited cutaneous SSc and the dcSSc subgroup. However, only patients with ILD 


\begin{tabular}{|c|c|c|c|c|}
\hline \multirow[t]{2}{*}{ TABLE 5} & \multicolumn{4}{|c|}{$\begin{array}{l}\text { Baseline characteristics in patients with and } \\
\text { without lung carbon monoxide diffusing capacity } \\
\text { decline during follow-up\#: univariate analysis }\end{array}$} \\
\hline & & Decline & No decline & $\mathrm{p}$-value \\
\hline \multicolumn{2}{|c|}{ Subjects $\mathrm{n}$} & 39 & 59 & \\
\hline \multicolumn{2}{|c|}{ Abn oesophageal manometry } & $33(84.6)$ & $41(69.5)$ & 0.18 \\
\hline \multicolumn{2}{|c|}{ LOS pressure $<10 \mathrm{mmHg}$} & $22(56.4)$ & $27(45.8)$ & 0.79 \\
\hline \multicolumn{2}{|c|}{ Aperistalsis } & $21(53.8)$ & $12(20.3)$ & 0.02 \\
\hline \multicolumn{2}{|c|}{ Hurwitz stage $\geqslant 3$} & $27(69.2)$ & $24 / 58(41.4)$ & 0.009 \\
\hline \multicolumn{2}{|c|}{ Hurwitz stage 4} & $16(41.0)$ & $9(15.3)$ & 0.06 \\
\hline \multicolumn{2}{|c|}{ Baseline FVC $<75 \%$ pred } & $4(10.3)$ & $10(16.9)$ & 0.85 \\
\hline \multicolumn{2}{|c|}{ SSc duration yrs } & $2(0-4)$ & $1(0-5)$ & 0.30 \\
\hline \multicolumn{2}{|c|}{ Anti-topoisomerase I } & $10(25.6)$ & $14(23.7)$ & 0.06 \\
\hline \multicolumn{2}{|c|}{ Diffuse cutaneous SSc } & $14(35.9)$ & $24(40.7)$ & 0.56 \\
\hline \multicolumn{2}{|c|}{ Overall aggravation of SSc } & $14(35.9)$ & $18(30.5)$ & 0.59 \\
\hline
\end{tabular}

were recruited in the SLS, whereas consecutive patients with and without ILD were included in the present study. Thus the SLS was not designed to compare the prevalence and outcome of ILD in the two cutaneous subtypes of the disease.

The SLS [6] and a recent study by GoH et al. [28] identified two principal predictors of progression to restrictive lung disease in SSc: high fibrosis extension score on initial CT scan [6]; and baseline FVC, the 70\%-pred threshold being most strongly associated with pulmonary prognosis [29]. In the present cohort, the extension of ILD was not assessed on CT scans, and the findings did not identify baseline FVC as a predictor of pulmonary function deterioration. The $70 \%$-pred threshold seemed to be associated with FVC decline on univariate analysis, but did not remain significant on multivariate analysis. This finding is probably due to too few patients having a baseline FVC of $<70 \%$ pred in the present cohort.

Finally, a poorer pulmonary outcome has previously been reported for black people [2, 28], males [28] and those of greater age [28], whereas smoking does not seem to be correlated with mortality in SSc patients [28]. The small number of patients in these subgroups limited the statistical power of the analyses; it was not possible to draw firm conclusions on this point.

The present study has several limitations. First, the systematic treatment of the patients with PPIs may have masked the consequences of GOR in the lung. However, a high frequency of oesophageal mucosal abnormalities has previously been reported in SSc patients on long-term PPI treatment [29], demonstrating the persistence of severe GOR. The pulmonary consequences of GOR, if there are any, might well have been observed despite this treatment. Moreover, it would have been unethical not to treat the patients with PPIs for a long period, given the severe digestive complications of GOR [14]. Thus the present data represent the real outcome of SSc in patients. Follow-up data regarding nonsteroidal anti-inflammatory drugs and prokinetics were, unfortunately, not available and may account for outcomes. A second limitation concerns the lack of systematic assessment of ILD on CT scans, but a comparison between PFT and CT scan outcomes was performed in half of the patients and showed reasonably good concordance between the two methods. Of the patients, $4 \%$ received intravenous cyclophosphamide for $\geqslant 6$ months. This may have affected the results of the study. Nevertheless, the number of patients concerned is very small, such treatment has not been demonstrated to be effective for this complication and PFT outcome was no better in these patients than in the other patients. Another limitation is the lack of assessment of 24-h intra-oesophageal $\mathrm{pH}$ recording and mucosal damage that should be evaluated in the future to more closely determine the role of GOR. The present study has also several strengths. Its prospective design limited bias. Consecutive patients were recruited, ensuring that the cohort was representative of the SSc population. The number of patients recruited, the $6 \mathrm{yrs}$ of follow-up and the multivariate analysis guarantee greater statistical power than in previous studies assessing the potential link between manometric findings and ILD.

\section{Conclusion}

In this prospective longitudinal study assessing predictive factors for pulmonary function deterioration in SSc patients, and the potential role of oesophageal involvement, the dcSSc subtype was the only significant predictor identified. These results do not support previous data suggesting a causative role of oesophageal involvement in SSc-ILD. Further studies are required in order to better establish the relative weights of each of the prognostic factors for SSc-ILD identified to date in larger studies.

\section{STATEMENT OF INTEREST}

A statement of interest for A.T. Dinh-Xuan can be found at www.erj. ersjournals.com $/ \mathrm{misc} /$ statements.dtl

\section{REFERENCES}

1 Schurawitzki H, Stiglbauer R, Graninger W, et al. Interstitial lung disease in progressive systemic sclerosis: high-resolution CT versus radiography. Radiology 1990; 176: 755-759.

2 Steen VD, Conte C, Owens GR, et al. Severe restrictive lung disease in systemic sclerosis. Arthritis Rheum 1994; 37: 1283-1289.

3 Steen VD, Medsger TA. Changes in causes of death in systemic sclerosis, 1972-2002. Ann Rheum Dis 2007; 66: 940-944.

4 Mayes MD. Scleroderma epidemiology. Rheum Dis Clin North Am 2003; 29: 239-254.

5 Allanore Y, Avouac J, Wipff J, et al. New therapeutic strategies in the management of systemic sclerosis. Expert Opin Pharmacother 2007; 8: 607-615.

6 Tashkin DP, Elashoff R, Clements PJ, et al. Cyclophosphamide versus placebo in scleroderma lung disease. N Engl J Med 2006; 354: 2655-2666.

7 Tashkin DP, Elashoff R, Clements PJ, et al. Effects of 1-year treatment with cyclophosphamide on outcomes at 2 years in scleroderma lung disease. Am J Respir Crit Care Med 2007; 176: 1026-1034.

8 Tobin RW, Pope CE 2nd, Pellegrini CA, et al. Increased prevalence of gastroesophageal reflux in patients with idiopathic pulmonary fibrosis. Am J Respir Crit Care Med 1998; 158: 1804-1808.

9 Schachter LM, Dixon J, Pierce RJ, et al. Severe gastroesophageal reflux is associated with reduced carbon monoxide diffusing capacity. Chest 2003; 123: 1932-1938. 
10 Lock G, Pfeifer M, Straub RH, et al. Association of esophageal dysfunction and pulmonary function impairment in systemic sclerosis. Am J Gastroenterol 1998; 93: 341-345.

11 Marie I, Dominique $S$, Levesque $\mathrm{H}$, et al. Esophageal involvement and pulmonary manifestations in systemic sclerosis. Arthritis Rheum 2001; 45: 346-354.

12 Savarino E, Bazzica M, Zentilin P, et al. Gastro-esophageal reflux and pulmonary fibrosis in scleroderma: a study using $\mathrm{pH}$-impedance monitoring. Am J Respir Crit Care Med 2009; 179: 408-413.

13 Troshinsky MB, Kane GC, Varga J, et al. Pulmonary function and gastroesophageal reflux in systemic sclerosis. Ann Intern Med 1994; 121: 6-10.

14 Wipff J, Allanore Y, Soussi F, et al. Prevalence of Barrett's esophagus in systemic sclerosis. Arthritis Rheum 2005; 52: 2882 2888.

15 Medsger TA Jr. Natural history of systemic sclerosis and the assessment of disease activity, severity, functional status, and psychologic well-being. Rheum Dis Clin North Am 2003; 29: 255-273.

16 Hurwitz AL, Duranceau A, Postlethwait RW. Esophageal dysfunction and Raynaud's phenomenon in patients with scleroderma. Am J Dig Dis 1976; 21: 601-606.

17 Ioannidis JP, Vlachoyiannopoulos PG, Haidich AB, et al. Mortality in systemic sclerosis: an international meta-analysis of individual patient data. Am J Med 2005; 118: 2-10.

18 Wells AU, Hansell DM, Rubens MB, et al. Fibrosing alveolitis in systemic sclerosis. Bronchoalveolar lavage findings in relation to computed tomographic appearance. Am J Respir Crit Care Med 1994; 150: 462-468

19 Goldin JG, Lynch DA, Strollo DC, et al. High-resolution CT scan findings in patients with symptomatic scleroderma-related interstitial lung disease. Chest 2008; 134: 358-367.
20 Lock G, Holstege A, Lang B, et al. Gastrointestinal manifestations of progressive systemic sclerosis. Am J Gastroenterol 1997; 92: 763-771.

21 de Souza RB, Borges CT, Capelozzi VL, et al. Centrilobular fibrosis: an underrecognized pattern in systemic sclerosis. Respiration 2009; 77: 389-397.

22 Limburg AJ, Beekhuis H, Smit AJ, et al. Esophageal hypomotility in primary and secondary Raynaud's phenomenon: comparison of esophageal scintigraphy with manometry. J Nucl Med 1995; 36: 451-455.

23 Bassotti G, Battaglia E, Debernardi V, et al. Esophageal dysfunction in scleroderma: relationship with disease subsets. Arthritis Rheum 1997; 40: 2252-2259.

24 Yarze JC, Varga J, Stampfl D, et al. Esophageal function in systemic sclerosis: a prospective evaluation of motility and acid reflux in 36 patients. Am J Gastroenterol 1993; 88: 870-876.

25 Richter JE. Oesophageal motility disorders. Lancet 2001; 358 823-828.

26 Ebina $M$, Shimizukawa $M$, Shibata $N$, et al. Heterogeneous increase in CD34-positive alveolar capillaries in idiopathic pulmonary fibrosis. Am J Respir Crit Care Med 2004; 169: 1203-1208.

27 Clements PJ, Roth MD, Elashoff R, et al. Scleroderma Lung Study (SLS): differences in the presentation and course of patients with limited versus diffuse systemic sclerosis. Ann Rheum Dis 2007; 66: 1641-1647.

28 Goh NS, Desai SR, Veeraraghavan S, et al. Interstitial lung disease in systemic sclerosis: a simple staging system. Am J Respir Crit Care Med 2008; 177: 1248-1254.

29 Marie I, Ducrotte P, Denis P, et al. Oesophageal mucosal involvement in patients with systemic sclerosis receiving proton pump inhibitor therapy. Aliment Pharmacol Ther 2006; 24: 15931601. 